\title{
Wavelet-based Optical Flow Analysis (wOFA) for Background Oriented Schlieren (BOS) Image Processing
}

\author{
B. E. Schmidt* \\ Case Western Reserve University, Cleveland, Ohio, 44106 \\ M. R. Woike ${ }^{\dagger}$ \\ NASA Glenn Research Center, Cleveland, Ohio, 44135
}

\begin{abstract}
A wavelet-based optical flow analysis (wOFA) method for processing background oriented schlieren (BOS) images is presented and demonstrated on synthetic and experimental data. Optical flow is inherently well-suited to BOS, since the background pattern and lighting conditions are specified and controlled by the user, and can be chosen to play to the strengths of optical flow processing. Analysis of the synthetic BOS data show that a 2D sinusoidal background produces the highest reconstruction accuracy for both wOFA and iterative least squares (ILS) algorithms. wOFA outperforms ILS in terms of overall accuracy for displacement fields with sufficiently high spatial frequency content. In addition, wOFA provides higher spatial resolution, about an order of magnitude in terms of the total number of pixels in the final BOS image. Finally, wOFA is demonstrated on two sets of experimental data, a heat gun plume experiment with nearly ideal imaging characteristics, and experiments in a supersonic wind tunnel flow with more realistic restrictions on the acquisition of images. BOS images computed with wOFA are shown to have higher spatial resolution and sensitivity than ILS, without introducing additional noise. Therefore, wOFA of BOS images are able to reveal flow features not detected by ILS analysis.
\end{abstract}

\section{Nomenclature}

$\underline{d}=$ displacement in the image plane

$\delta \quad=\quad$ specified displacement

$\epsilon_{\mathrm{RMS}}=$ RMS error

$\varepsilon \quad=$ deflection angle

$I_{d} \quad=$ data image

$I_{r} \quad=$ reference image

\footnotetext{
*Assistant Professor, Mechanical and Aerospace Engineering, 2123 Martin Luther King Jr. Drive, AIAA Senior Member. ${ }^{\dagger}$ Research Engineer, Optics and Photonics Branch, 21000 BrookPark Road, AIAA Senior Member.
} 


$$
\begin{array}{ll}
K & =\text { Gladstone-Dale constant } \\
N & =\text { number of pixels } \\
n & =\text { index of refraction } \\
\rho & =\text { density } \\
\sigma & =\text { standard deviation } \\
x, y & =\text { spatial coordinates } \\
\psi & =\text { wavelet transform of } \underline{d} \\
s & =\text { coordinate along optical path } \\
\Omega & =\text { image domain }
\end{array}
$$

\section{Introduction}

Background oriented schlieren (BOS) is a relatively new technique for visualizing refractive index gradients in transparent media. BOS has several advantages compared to a conventional schlieren system, including a simpler and more adaptable setup and the potential for performing three-dimensional imaging and extracting quantitative information about the refractive index field [1, 2]. A comprehensive review of BOS theory and its applications is given by Raffel [3], and a more succinct review that places BOS in context with other related visualization techniques is given by Settles and Hargather [4]. A schematic of a typical BOS setup is shown in Fig. 1] A camera is focused on a patterned background, and a reference image $I_{r}$ is acquired with no phase disturbances between the camera and the background. Light rays propagating from the background to the camera are refracted by an angle $\varepsilon$ as they pass through a field with varying index of refraction $n$ according to

$$
\varepsilon_{x}=\frac{1}{n_{0}} \int \frac{\partial n}{\partial x} \mathrm{~d} s, \quad \varepsilon_{y}=\frac{1}{n_{0}} \int \frac{\partial n}{\partial y} \mathrm{~d} s
$$

where $n_{0}$ is the index of refraction in the undisturbed medium, $s$ is the variable along the propagation path and the gradient is taken in a plane orthogonal to the optical path. Assuming small deflection angles and paraxial rays, the displacement of a point on the background pattern in the imaging plane $(\Delta x, \Delta y)$ is given by

$$
\Delta x=Z_{D} M \varepsilon_{x}, \quad \Delta y=Z_{D} M \varepsilon_{y}
$$

where $M$ is the image magnification, $M=\frac{Z_{i}}{Z_{A}+Z_{D}}$. These displacements are typically in the sub-pixel range, and usually no larger than 1-2 pixels. A data image $I_{d}$ is acquired with the background image distorted by the phase disturbances. The local displacement between $I_{r}$ and $I_{d}$ is representative of the change in $n$ along the optical path according to Eqs. (1) and (2). 


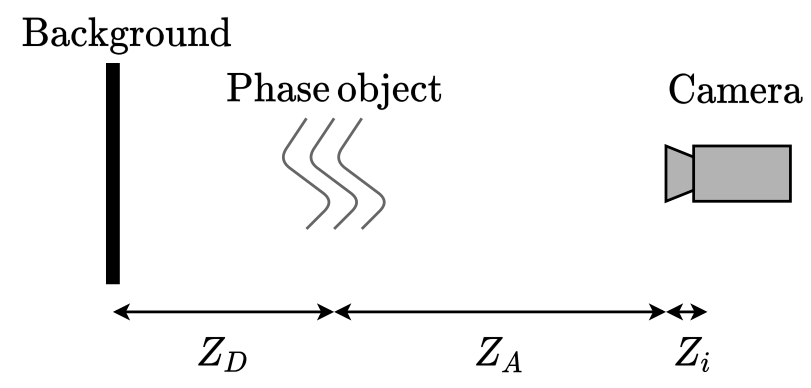

Fig. 1 A typical BOS setup, viewed from the side. $Z_{D}$ is the distance from the phase object to the background, $Z_{A}$ is the distance from the phase object to the camera lens, and $Z_{i}$ is the distance from the camera lens to the sensor.

In a gas, the index of refraction $n$ is linearly related to the density via the Gladstone-Dale relation,

$$
n=K \rho+1,
$$

where $K$ is the Gladstone-Dale constant for the gas. $K$ is also a weak function of the wavelength of the light passing through the gas. Equation (3) allows the gradients in $n$ visualized with BOS (or conventional schlieren) to be interpreted as gradients in $\rho$. This makes BOS a very useful tool for analyzing flows with variable density, including compressible flows and flows with chemical reactions.

Once the reference and data images $I_{r}$ and $I_{d}$ have been acquired, the remaining task in BOS is to determine the displacement field $\underline{d}(\underline{x})$ between the images. By far the most common approach is to use either a cross-correlation or iterative least squares (ILS) algorithm, like those used for particle image velocimetry (PIV) and digital image correlation (DIC), respectively [3]. An abbreviated list of examples of studies using these algorithms can be found in refs. [5]-9]. ILS algorithms are beginning to be favored over cross-correlation due to increased accuracy in determining sub-pixel displacements. The primary disadvantage of PIV or DIC algorithms is a loss in resolution between the acquired images $I_{r}$ and $I_{d}$ and the computed displacement field $\underline{d}$. This is due to the use of multi-pixel interrogation windows in both types of algorithms [10, 11]. Interrogation windows are regions, typically ranging between $16 \times 16$ and $64 \times 64$ pixels for PIV and smaller for DIC, that are compared between $I_{r}$ and $I_{d}$. The interrogation windows must contain enough spatial information to produce a sufficiently strong correlation peak, or match in the least-squares sense, to produce a valid displacement vector. For PIV applications, the common rule of thumb is that each interrogation window must contain at least 7 tracer particles [12]. The requirement is less clear for DIC or BOS where the background pattern may not contain discrete particle-like images. Application of PIV/DIC algorithms produces one displacement vector per interrogation window. The windows can be overlapped to increase spatial resolution, but there is still typically a loss in resolution of about an order of magnitude in terms of the total number of displacement vectors. This can lead to the blurring or loss of sharp features in the image. 
One recent alternative to PIV- or DIC-like processing that can produce higher resolution BOS images is dot tracking, similar to methods used for particle tracking velocimetry (PTV) [13]. Dot tracking methods restrict users to backgrounds that are made up of discrete particle-like images, however, and they have been shown to be more sensitive to imaging noise than cross-correlation, although still more accurate at high levels of noise. Furthermore, they are computationally more expensive than ILS or cross-correlation algorithms.

Another approach is to employ optical flow analysis (OFA) to calculate the displacements. Optical flow is widely used in the computer vision community to determine the motion of objects in a scene using successive frames in a video sequence. Since the seminal work of Horn and Schunck [14], a multitude of optical flow algorithms have been created and applied to a wide variety of problems. Optical flow algorithms produce displacement fields with the same spatial resolution as the original images, i.e. one vector per pixel. OFA was first applied to BOS processing by Atcheson et al. [15], who applied three different optical flow algorithms [14, 16, 17] to a set of synthetic BOS images in order to accurately quantify the error of each. They found that the optical flow algorithms outperformed a correlation-based algorithm not only in terms of spatial resolution, but also in terms of the overall accuracy. Somewhat surprisingly, little progress has been made in terms of the application of OFA to BOS, and only a handful of studies have been published that employ OFA [15, 18, 26].

In this work, a recent optical flow algorithm originally developed for velocimetry [27, 28] is adapted for application to BOS. As opposed to classical OFA approaches, the algorithm in this work is known as a wavelet-based optical flow analysis (wOFA) method because it solves for the displacement in the wavelet domain assuming a level of sparsity in the wavelet coefficients. The organization of the paper is as follows. First, the wOFA method is described in detail. Second, some new, automated image pre-processing routines for mask generation and camera shift correction for BOS are given. These are not unique to wOFA approaches, but can be generally applied before BOS processing with any algorithm. The wOFA method is then applied to a set of simulated image data for various backgrounds, similar to the approach of Atcheson et al. [15], and the results are compared to those from a commercial, state-of-the-art ILS method. Finally, results from applying the wOFA method to two sets of experimental data are presented and compared against results from ILS.

\section{Optical Flow Method}

Optical flow methods in general rely on the pixel intensity being conserved between a pair of images in a sequence or, in this case, between the $I_{r}$ and $I_{d}$. This is expressed via the displaced frame difference (DFD) equation,

$$
I_{r}(\underline{x})-I_{d}(\underline{x}+\underline{d}(\underline{x}))=0
$$


Equation (4) is expressed as an advection equation in gradient-based approaches such as that of Horn and Schunck [14] by invoking the Taylor expansion of spatial derivatives,

$$
\frac{\partial I(\underline{x}, t)}{\partial t}+\underline{d}(\underline{x}, t) \cdot \underline{\nabla} I(\underline{x}, t)=0
$$

but it has been shown that variational approaches which solve Eq. (4) directly are more accurate and can handle larger displacements [17]. Part of the increase in accuracy is due to the elimination of the need to compute finite-difference approximations to derivatives in Eq. [5], which can amplify imaging noise. In these approaches, a warping operator is applied to $I_{d}$ in order to compute $I_{d}(\underline{x}+\underline{d}(\underline{x}))$. Most often, the displacement $\underline{d}$ is calculated by finding a the value $\underline{d}=\underline{\hat{d}}$ that minimizes a penalty function:

$$
\underline{\hat{d}}=\underset{\underline{d}}{\operatorname{argmin}} J_{\mathrm{D}}\left(I_{r}, I_{d}, \underline{d}\right)+\lambda J_{\mathrm{D}}(\underline{d}) .
$$

$J_{\mathrm{D}}$ is a penalty term based on Eq. (4). The present methods uses a Lorentzian penalty function, which is a "softer" penalty function than the more commonly used quadratic penalty, meaning it is less sensitive to outliers [29]. $J_{\mathrm{R}}$ is a regularization term that enforces smoothness on the calculated displacement field, and $\lambda$ is a scalar parameter that balances the two terms. $\lambda$ is currently determined empirically for a given BOS experiment based on the appearance of the BOS images, but typical values are in the range $-3<\log _{10} \lambda<-0.5$. Larger values of $\lambda$ lead to smoother solutions and can cause over-smoothing of the estimated displacement field, but smaller values tend to enhance noise in the BOS result. In classical optical flow approaches, a regularization term is also required to stabilize the ill-posed minimization problem in Eq. 6. Many OFA methods in the literature use a Horn and Schunck regularizer, equivalent to first-order Tikhonov regularization,

$$
J_{\mathrm{R}}=\int_{\Omega}\left\|\underline{\nabla} d_{x}\right\|^{2}+\left\|\underline{\nabla} d_{y}\right\|^{2} \mathrm{~d} x \mathrm{~d} y,
$$

where $\Omega$ represents the image domain. While suitable for rigid motions, several researchers have shown in OFV applications that Eq. (7) produces over-smoothing unsuitable for representing fluid motion [30-32]. Even in image processing applications, first-order Tikhonov regularization can produce undesirable over-smoothing of edges and the production of non-physical artifacts [33]. For these reasons, in this work $J_{\mathrm{R}}$ instead penalizes the Laplacian of the displacement field,

$$
J_{\mathrm{R}}=\int_{\Omega}\left\|\underline{\nabla}^{2} \underline{d}\right\|^{2} \mathrm{~d} x \mathrm{~d} y .
$$

Laplacian smoothing is used extensively in image processing for smoothing while preserving sharp edges and features [34].

Unlike classical OFA approaches, wOFA methods do not solve for the components of $\underline{d}$ directly. Instead, Eq. (6) 
takes the form

$$
\underline{\hat{d}}=\underset{\underline{\psi}}{\operatorname{argmin}} J_{\mathrm{D}}\left(I_{r}, I_{d}, \underline{d}\right)+\lambda J_{\mathrm{R}}(\underline{\psi}),
$$

where $\underline{\psi}$ is the wavelet transform of $\underline{d}$. As in [28], separable, biorthogonal 17-11 Cohen-Daubechies-Favreau wavelets with symmetric boundary conditions are used. As described in [27], Eq. 9] is solved assuming that the finest scale wavelet coefficients in $\underline{\psi}$ are zero. This reduces the number of unknowns by a factor of four compared to Eq. [6, and the minimization problem is therefore no longer ill-posed, meaning that numerical stability is achieved even without an explicit regularization term. Truncation of the wavelet coefficients at the finest scale does not lead to any appreciable inaccuracy as long as the images are reasonably well-resolved due to the natural smoothness of density fields in fluids (see Fig. 1 of 27). A non-zero value of $\lambda$ in wOFA serves only to add smoothness to the solution and enhance accuracy by reducing noise. Additionally, no explicit multi-scale scheme is needed to estimate large displacements, because the coefficients of $\underline{\psi}$ are solved for sequentially, from coarse scales to fine scales. For more information on the coarse-to-fine strategy, see [28].

An additional advantage of wOFA approaches over classical OFA methods is that regularization can be implemented in the wavelet domain in a stable, efficient way without the need to solve the Euler-Lagrange equations for $J_{R}$. This is done by applying differentiation to the smooth scaling functions of the wavelet, which can be performed analytically without the use of finite difference schemes as shown by Beylkin [35]. The wavelet transforms and regularization are performed with the matrix factorization approach developed by Schmidt and Sutton [28].

In order to handle images that are non-square and/or have dimensions that are not a power of two, the images are divided into square sub-domains or "patches" that have a size of a power of two, e.g. $256 \times 256$ or $512 \times 512$ pixels. The patches are overlapped by at least $10 \%$ on each side and cover the entire image domain. After computing the displacement for every patch, a separable, two-dimensional Nuttall window [36] is applied to each patch during reconstruction to mitigate edge effects near the patch boundaries.

\section{Image Pre-processing}

In many cases, pre-processing steps must be applied to $I_{r}$ and $I_{d}$ prior to computing the displacement. A simple brightness correction and linear contrast enhancement is applied to both images to compensate for any unstable lighting, which would violate Eq. (47. In addition, two other effects frequently occur in BOS experiments that require special attention. The first is the relative motion of the camera and the background pattern between the reference and data images due to vibration or other causes. This causes an apparent bulk motion of the background pattern, which would make an undesirable contribution to $\underline{d}$.

The second is obstruction of the background by solid object(s), such as a wind tunnel model. Comparison of the image of the solid object to the background in the reference image is meaningless, and furthermore can destabilize 
cross-correlation and ILS algorithms near the model surface. Furthermore, for wOFA approaches, computing the displacement on the pixels which contain solid object can corrupt the displacements computed in the neighborhood of the object because of the finite width of the wavelet filters, rendering them useless. This issue also frequently arises in PIV experiments, and the solution is to apply a logical mask digitally over the solid objects, where velocity, or displacement in the case of BOS, is not computed. Most often, the mask location is specified manually, although some automated, dynamic masking techniques have been developed for PIV, most of which use statistical or edge-finding methods to determine the mask location [37, 38]. To the author's knowledge, however, no automated mask generation strategy has been developed specifically for BOS.

\section{A. Camera Shift Correction}

Correcting for camera shift, or relative motion between the camera and background, is an image registration problem. Image registration is a widely-studied topic in computer vision, and a multitude of solution algorithms exist [39]. In general, image registration techniques typically compensate for translation, rotation, scaling, and shear, or any combination of those effects. In this work, the camera shift is assumed to consist only of translation, but other effects could be included if necessary without much additional computational cost. In the present work, it is recognized that image registration and optical flow are closely related problems in inverse image processing. Both problems, in essence, seek to find a mapping that will match one image to another, typically by minimizing some function of the difference in pixel intensities between the two images. Therefore, wOFA is used to correct for the camera shift instead of employing a separate image registration tool.

The procedure begins with the user specifying a region, called the anchor region, in both $I_{r}$ and $I_{d}$ in which there are no distortions due to optical index variation. In this way, the only displacement in the anchor region is due to relative motion between the camera and the background. If it is not possible to find such a region, it is necessary to make an assumption about the average refractive index gradient in a region of the image, for example that the average gradient is zero. Next, $\underline{d}$ is computed for the anchor region using the wOFA method described in Sec. II with a large value of $\lambda$ $(\lambda=1)$, because fine scale motion is not important. The camera shift is then specified to be the mean of the calculated displacement for the entire anchor region, since the shift is assumed to be a result of translation only. The calculated camera shift is applied to the entire image prior to computing the BOS image. The shift correction procedure in the present work is observed to perform well for shifts of less than about 10 pixels.

\section{B. Automatic Masking}

To the authors' knowledge, all BOS methods capable of processing with a logical mask require that the mask location be specified manually. In certain cases, such as when the obstructing object has a simple geometric shape and does not move during a test sequence of multiple data images, manual masking does not present many difficulties as it can be 
performed easily once for all data images. Many situations, however, feature models with complex geometry that are not easily masked manually. Furthermore, the model may be in free flight [20, 22], or move slightly due to aerodynamic loading during a test, meaning that a unique mask must be computed for each data image. In sequences of hundreds or even thousands of data images, this becomes an immense task if the masks must be computed manually.

An important feature of BOS images that can be exploited for automatic mask generation is a difference between the appearance of the background, which typically consists of a random, noise-like pattern, and the solid object, which has a much smoother texture. The masking routine developed in the present work uses this difference in features to generate a logical mask according to the following steps.

1) A median filter with a large kernel, $21 \times 21$ pixels in the present method, is applied to the image $I$. The filter smooths the high-frequency pattern of the background but has little effect on the object(s) to be masked. The original image is then subtracted from the median-filtered image, highlighting the background pattern. This methodology is similar to that of Kiger and Pan [40], who used median filtering and subtraction to perform size discrimination of particles in multiphase flows.

2) The image from step 1 is then processed with a log-of-Gaussian (LoG) filter to detect edges. Edges appear as thin, logical contours in the region with the background pattern, but are not detected in the foreground object(s) because of the subtraction of those regions in step 1.

3) The image from step 2 is then blurred with a Gaussian filter $(\sigma=8)$ to blend the edges identified in the background pattern together.

4) An edge-aware Laplacian filter [34] is applied to the resulting image from step 3 that smooths the background pattern and foreground object(s), but leaves the edge between the regions sharp. At this stage, the image intensity is approximately zero on foreground objects and has non-zero, positive values on the background pattern.

5) Finally, the image from step 4 is thresholded to convert to a logical mask, with logical 1 on the background pattern and logical 0 on the foreground object(s) to be masked out.

The masking routine is applied to both the reference and data images, and the calculated masks from both images are combined to form a composite mask when computing the displacement. The wOFA algorithm ignores masked regions when computing $J_{D}$ in Eq. 97, so masked regions do not contribute to the calculation of $\underline{d}$. In addition, large, continuous masked regions are ignored entirely during wOFA processing for enhanced computational efficiency.

An example image and the computed mask is shown in Fig. 2. The image is from a set of BOS experiments in a wind tunnel, and the wind tunnel model can be seen in Fig 2 a. The model moves slightly during a test run due to vibrations from aerodynamic loading, so a mask is computed for each data image. The masking routine described above was successfully applied to over a dozen data sets with different model geometries, cameras, and lighting conditions without the need to adjust parameters of the filters, indicating that it is sufficiently robust to be applied generally to any BOS data. The masking routine should be applied after any intensity and camera shift corrections, as the last step in 
image pre-processing.

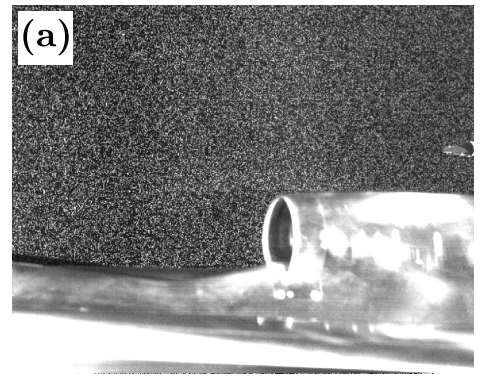

Fig. 2 (a) An example data image from a BOS experiment in a wind tunnel. The model, which should be masked prior to BOS computation, is in the lower region of the image. (b) The computed mask using the automated masking routine described in this section. The black region represents the mask, wOFA is only performed on the white region.

\section{Results and Discussion}

In this section, BOS results from the present wOFA method are examined for several sets of data and compared to a commercial, state-of-the-art ILS BOS algorithm. First, the algorithms are applied to a set of synthetic reference and data images with various background patterns and distortions in Sec. [V.A] in a manner similar to Atcheson et al. [15]. The use of synthetic data allows the error to be directly quantified, because the true displacement $\underline{d}_{\text {true }}$ is known a priori. Second, the algorithms are compared less quantitatively using two sets of experimental data in Sec. IV.B.

\section{A. Simulated Data}

Six background patterns are tested to study the effect of the background pattern on the performance of the BOS algorithms. For each background, a set of $20512 \times 512$-pixel reference images are generated to generate error statistics. Examples of the six types of background images are shown in Fig. 3. and listed below.

(a) 2D sinusoid with a period of 16 pixels. This pattern is highly regular and spatially repeating, which is discouraged for ILS BOS algorithms, but Grauer and Steinberg [24] found that it performs very well with OFA processing. The period was determined to be the best compromise in terms of accuracy for a range of spatial frequency in the displacement field.

(b) Gaussian noise pattern. This is a popular background for BOS experiments, and was examined by Atcheson et al. [15]. The images are produced by smoothing a random field with a Gaussian filter $(\sigma=1.75)$.

(c) Perlin noise [41]. This more complex noise pattern was originally designed to create realistic textures for computer graphics.

(d) Pseudo-PIV. The image simulates a PIV image acquired with a high seeding density, generated using the method described in [42].

(e) Random dot pattern. This is one of the most common patterns used in BOS experiments, and was examined by 
both Atcheson et al. [15] and Grauer and Steinberg [24].

(f) Wavelet noise [43]. This is a more advanced version of Perlin noise developed by animators at Pixar to generate realistic, multi-scale textures in computer animations. It was also shown to have good performance for BOS processing by Atcheson et al. [15]
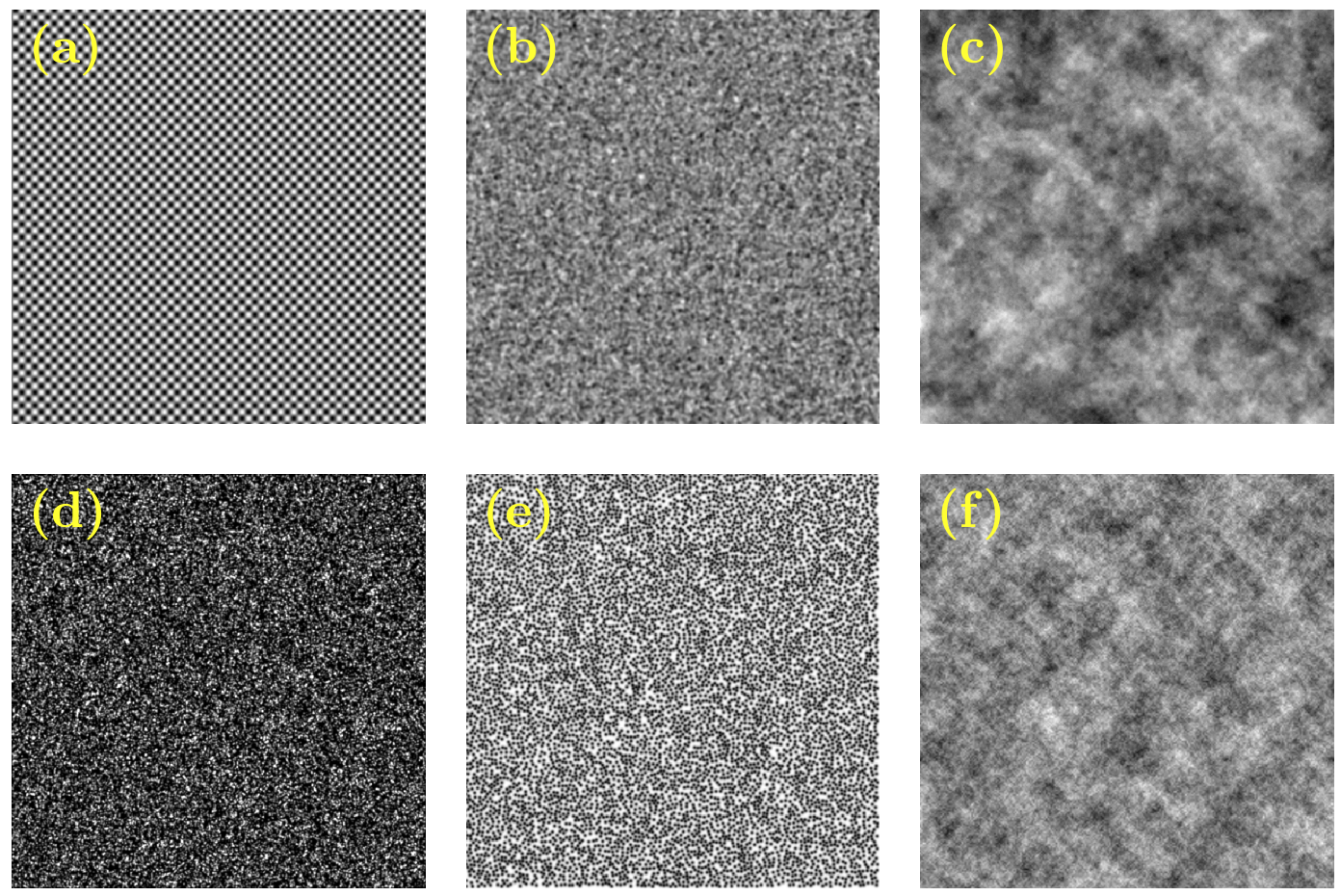

Fig. 3 Synthetic background patterns studied in the present work. (a) 2D sinusoid. (b) Gaussian noise. (c) Perlin noise. (d) Pseudo-PIV. (e) Random dots. (f) Wavelet noise.

Each of the background images is distorted by a known displacement field $\underline{d}_{\text {true }}$ using a warping operator to create a data image. The displacement fields are random, isotropic distortions produced by first generating a random vector field with components drawn from the distribution $[-1,1]$. These vector fields contain unrealistic gradients for typical BOS images, so they are smoothed with a Gaussian filter. Three filter widths are used, $\sigma \in[5,11,23]$ pixels, to simulate features with different spatial frequency content. One component of example displacement fields for the three filter widths are shown in Fig. 4. The displacement magnitudes were varied, and sets with maximum displacements of 0.25 , $0.5,1,2$, and 4 pixels were generated. A set of 20 data images with a different random displacement field was generated for each combination of displacement magnitude and filter width, resulting in a total of $20 \times 20 \times 3 \times 5=6000$ image pairs for each of the six background patterns.

The RMS error for each calculated displacement field $\underline{\hat{d}}$ is computed and used to generate the statistics shown in Fig. 5.The RMS error is given by

$$
\epsilon_{\mathrm{RMS}}=\sqrt{\frac{1}{N} \sum\left\|\underline{\hat{d}}-\underline{d}_{\text {true }}\right\|^{2}}
$$



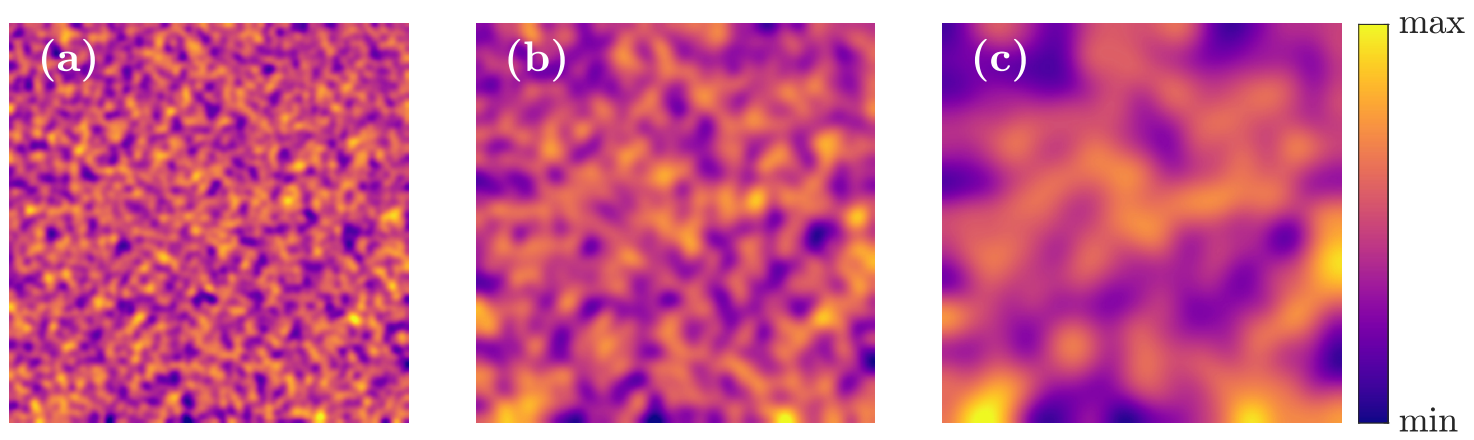

Fig. 4 One component of example synthetic displacement fields with filter widths of (a) 5, (b) 11, and (c) 23 pixels. The scale is arbitrary, it is only meant to demonstrate the spatial frequency content for each filter width.

where the summation is performed over all pixels in the image, excluding the image boundaries. Each data point represents the mean of the RMS error for a set of images, normalized by the maximum displacement, for each combination of background pattern, displacement magnitude, and spatial filter width for wOFA and ILS. The error bars are the standard deviation for each combination. The ILS computations were performed with a window and step size of 15 and 5 pixels, respectively, for each set besides the 2D sinusoid, which used a window and step size of 9 and 6 pixels, respectively. These parameters were optimal for the synthetic data. The first observation is that wOFA outperforms ILS
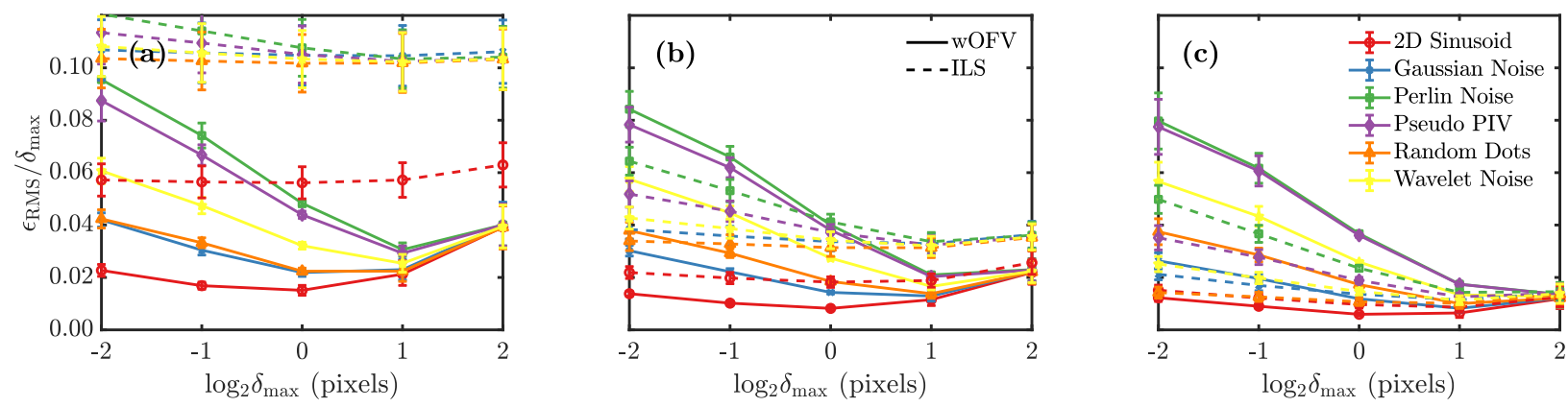

Fig. 5 Error results for each background pattern and BOS algorithm as a function of maximum displacement and spatial filter width: (a) 5, (b) 11, and (c) 23 pixels.

in terms of accuracy for almost every background pattern and displacement magnitude combination tested, and the gains in accuracy for wOFA are greater for displacement fields with higher spatial frequency content. This is to be expected, as it has already been noted that wOFA has significantly higher spatial resolution than ILS. Another finding is that the relative error of both wOFA and ILS is largely insensitive to the magnitude of the displacement field. This has important implications for experiments, where there is a trade-off between the displacement magnitude and the image quality [3].

Finally, it is observed that the three best background patterns for both wOFA and ILS are the 2D sinusoid, Gaussian noise, and random dots. This is a somewhat unexpected result, both because the findings of Atcheson et al. [15] indicate that a complex, multi-scale noise pattern such as Perlin or wavelet noise would produce the best results, and because 
regular patterns such as the 2D sinusoid are not recommended for ILS. It is noted that the ILS method was very sensitive to processing parameters for the $2 \mathrm{D}$ sinusoid background, and many parameter combinations resulted in a failure to converge to a result, so it may be best to avoid a sinusoidal background in practice if an ILS algorithm is to be employed. wOFA, however, suffered no such stability issues for any of the background patterns, and the 2D sinusoidal background processed with wOFA produced the lowest error for every combination of displacement magnitude and spatial filter width. The analysis performed to generate Fig. 5 used ideal, noise-free images, but the analysis was repeated with blurring, via a Gaussian filter with a kernel of 0.75 pixels, and added random noise to simulate experimental imaging effects. The added noise was Gaussian with a standard deviation of 3 bits on a simulated 12-bit sensor, assuming the full dynamic range of the BOS images to be the central $75 \%$ of the sensor (bits 512-3583). Although the errors were slightly higher for each image pair for the images with blurring and noise, the trends and conclusions are essentially identical.

\section{B. Experimental Data}

Results from two BOS experiments are presented in this section. The images are processed with both wOFA and ILS. The error cannot be quantified as it could in Sec. IV.A because the true displacement field is not known, but a qualitative analysis of the BOS image quality still yields meaningful results. The example experiments presented here are representative of two types of BOS experiments. The first, in Sec. [IV.B.1] is a highly controlled experiment where the imaging arrangement, lighting, and other parameters can be chosen by the user with very few restrictions, resulting in images with very high quality. The second, in Sec.IV.B.2 is an experiment from a wind tunnel that features many of the challenges commonly encountered by researchers, such as obstruction of the background by solid foreground objects, model vibration, and low image signal-to-noise ratio due to low illumination.

\section{Heat Gun Plume}

The first experiment is a plume from a heat gun with a random dot background pattern. The turbulent flow from the heat gun is at a higher temperature, and therefore lower density, than the ambient air, which creates variations in $n$ according to Eq. (3). Devices such as heat guns, candles, air dusters, and lighters are often used to characterize the performance of various types of schlieren systems, including BOS setups [44]. The calculated displacement from both wOFA (left column) and ILS (right column) are shown in Fig. 6. A mask was not needed for wOFA processing because the end of the heat gun, visible at the bottom of the images, did not negatively impact the calculation of the displacement field. One advantage of BOS imaging compared to conventional schlieren apparent in Fig. 6is that the optical index gradients in all directions are computed simultaneously, so the displacement in the horizontal ( $x$ ) (top row) and vertical (y) (middle row) directions can be viewed, along with the displacement magnitude (bottom row).

A comparison of the two columns in Fig. 6 indicates that the wOFA processing is able to resolve finer turbulent structures in the plume than ILS, which produces blurring of small features. In addition, a close examination of 

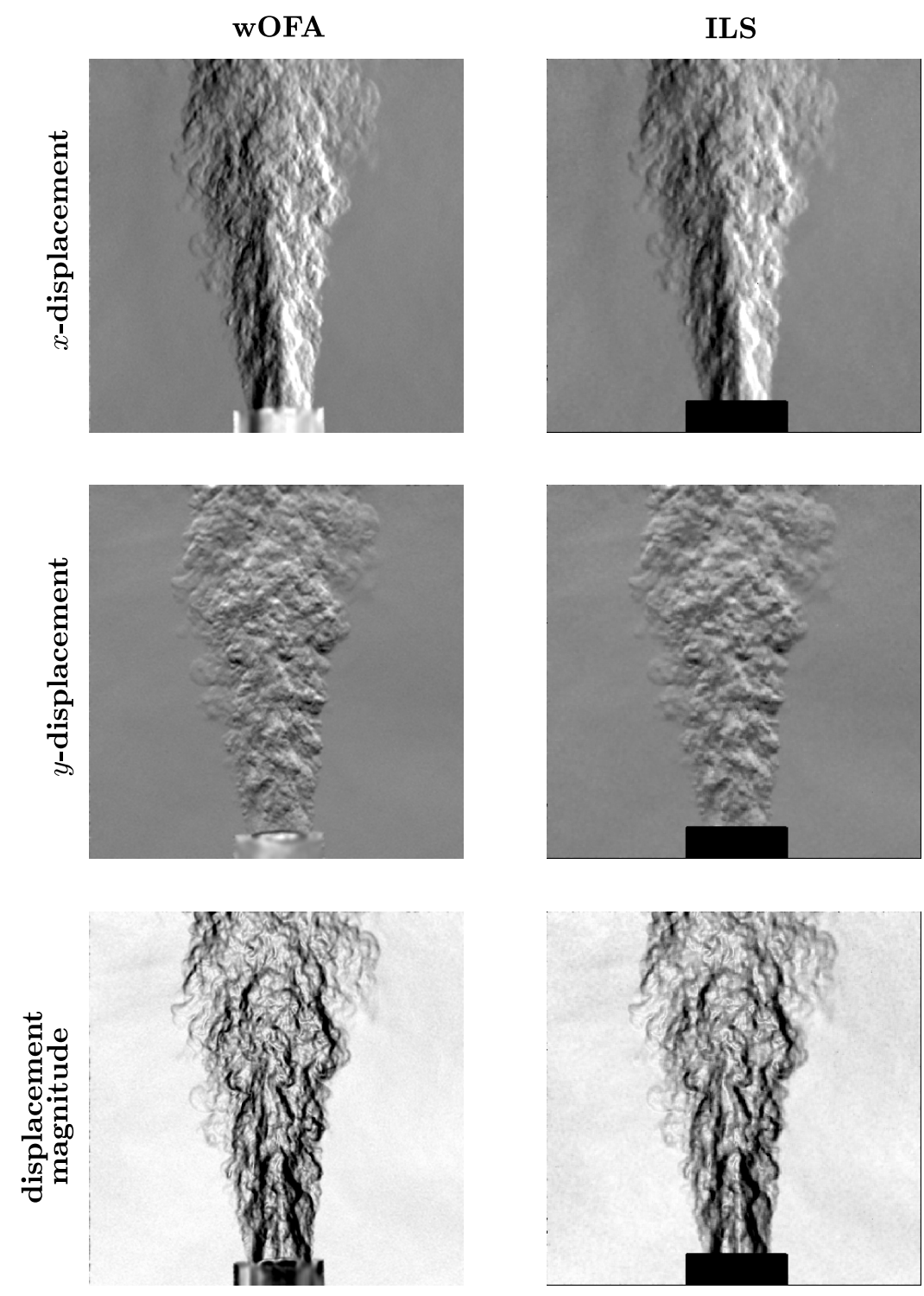

Fig. 6 Example BOS images of a heat gun plume processed with wOFA and ILS.

the pixel intensities in the BOS images suggests that wOFA is also more sensitive than ILS, and more accurately captures high-frequency fluctuations in the calculated displacement field. This is demonstrated more quantitatively in Fig.77, which shows the pixel intensity of the $y$-displacement along the heat gun plume centerline. These qualitative findings, higher accuracy in capturing fine-scale displacements and higher spatial resolution, align with the quantitative conclusions of Sec.IV.A. 


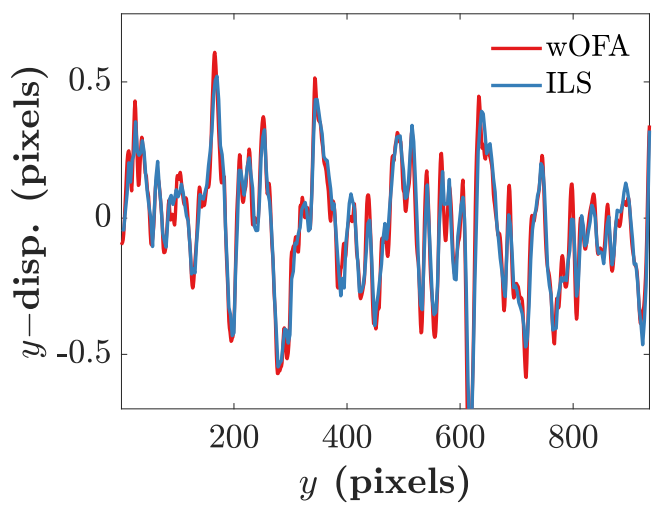

Fig. 7 Pixel intensity of the $y$-displacement along the centerline of the heat gun plume for wOFA and ILS.

\section{Supersonic Wind Tunnel Model}

The second experiment is a set of images from a test of a low-boom supersonic demonstrator model in the NASA Ames $11 \mathrm{ft}$. by $11 \mathrm{ft}$. transonic wind tunnel with a retroreflective background for improved light collection with low levels of illumination. More information about the model and details about the experimental arrangement can be found in Refs. [45], [21], and [46]. A total of 200 data images were acquired in supersonic flow. Density changes are associated with shock and expansion waves, as well as boundary layers, in compressible flows such as the one in this example, which produce changes in $n$, allowing them to be visualized with schlieren techniques. An example instantaneous BOS image as well as the mean image is shown for wOFA and ILS in Fig. 8 All four images in Fig. 8 show the optical index gradient in the vertical direction.

Masking was applied to the images prior to processing. The ILS images were produced with a manual mask specified for one data image and applied to every image in the sequence. Due to model vibrations, the mask was made larger than the model to ensure that the model was covered at all times. The wOFA results were produced using the automated mask generation procedure described in Sec. III.B Because the mask is generated for each individual data image, it more closely aligns to the model contour and allows for closer analysis of features such as the boundary layer on the model wall. Three horizontal bars, which are part of the wind tunnel infrastructure, also occlude some of the field of view and are masked for both processing methods.

Similar conclusions are drawn from the instantaneous images as for the heat gun data in Sec. IV.B.1 in terms of advantages of wOFA over ILS regarding spatial resolution and accuracy of resolving fine features. The noise in the wind tunnel prevents the shock pattern around the model from being resolved; however, it can be clearly observed in the mean images (bottom row of Fig. 87. wOFA again shows sharper definition of oblique shocks and expansion waves compared to ILS. In particular, wOFA is able to identify a set of weak waves propagating from the underside of the model, marked by a red ellipse in the lower left panel of Fig. 8 , that ILS cannot. Figure 9 shows this region of the wOFA and ILS images more closely so that it is easier to discern the features. The individual waves are blurred together in the 

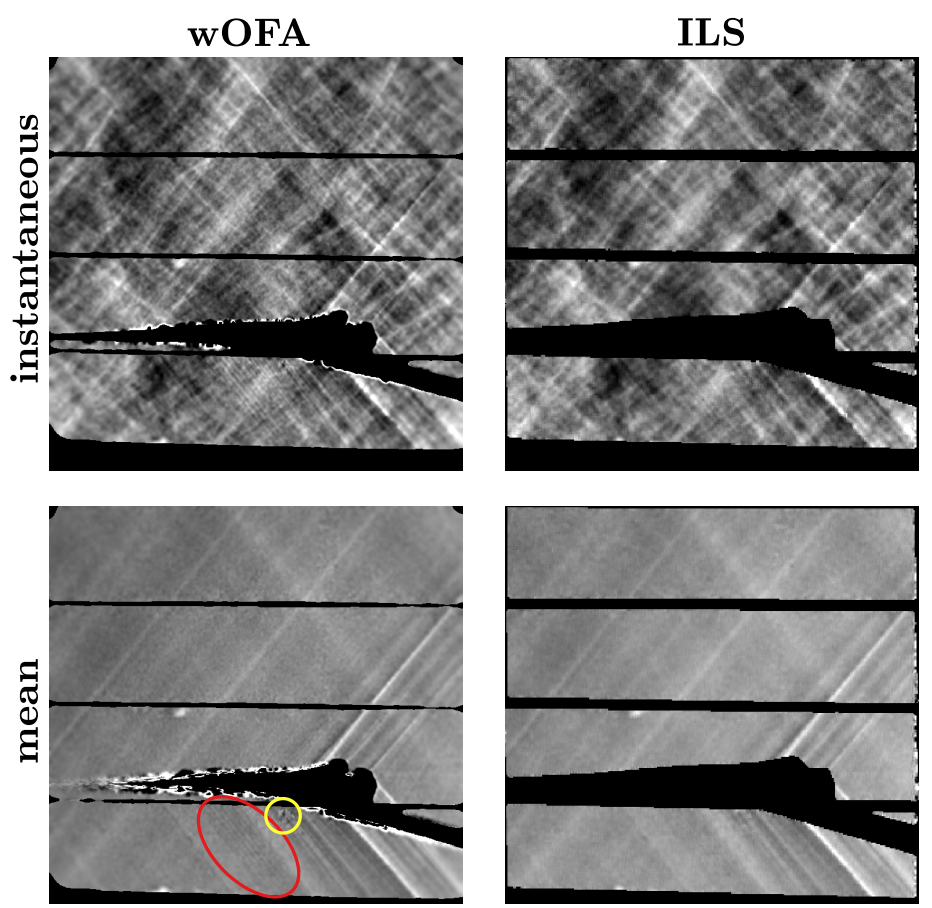

Fig. 8 Instantaneous (top row) and mean (bottom row) BOS images of the vertical optical index gradient computed with wOFA (left column) and ILS (right column) for the low-boom supersonic demonstrator wind tunnel model.

ILS result (right panel). These features can be observed in the instantaneous wOFA image as well (top left panel of Fig. 8), but their appearance in the mean image indicates that they result from a geometrical feature on the model, rather than from turbulent structures propagating in the model boundary layer, and they have been observed in previous studies on this geometry [21].
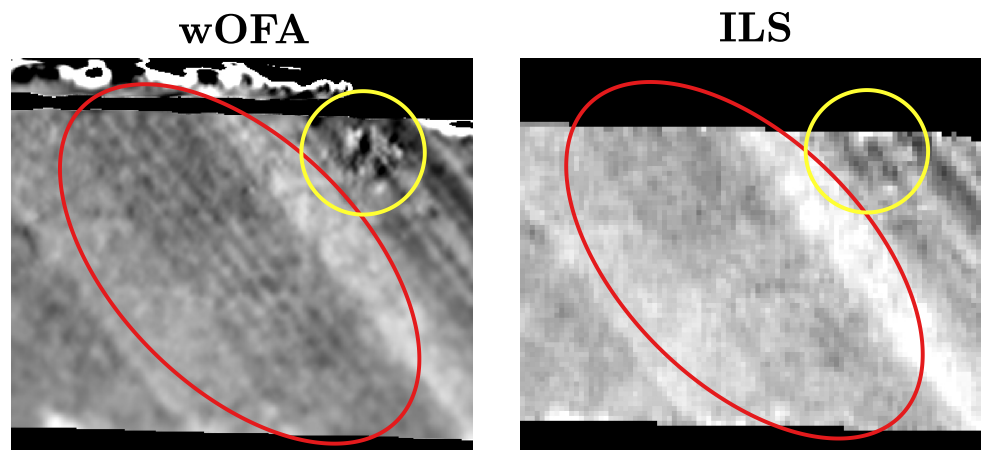

Fig. 9 Closeup view of the mean BOS images of the vertical optical index gradient computed with wOFA (left) and ILS (right) for the low-boom supersonic demonstrator wind tunnel model (bottom row of Fig. 8.

A second feature apparent in the wOFA results that does not appear in the ILS results is marked with a yellow circle in the left panel. Based on analysis of other data from the same experimental campaign, it is concluded that this structure is not a flow feature, but rather an imperfection in the background pattern, such as a bubble or tear, that causes 
an apparent displacement between the reference and data images. This finding represents a point of caution for wOFA processing: because wOFA is more sensitive to small and fine-scale displacements than ILS, more care must be taken to ensure that there are minimal disturbances to the experimental setup between acquisition of the reference and data images.

\section{Conclusions \& Future Work}

A wavelet-based optical flow analysis (wOFA) method for processing background oriented schlieren (BOS) images has been presented and demonstrated on synthetic and experimental data. In Sec. [III automated processing routines for correcting for relative motion between the camera and BOS background and masking foreground images were developed. Analysis of the synthetic BOS data revealed that (i) a 2D sinusoidal background produces the highest reconstruction accuracy for both wOFA and ILS algorithms, followed by Gaussian noise and a random dot pattern, and (ii) wOFA outperforms ILS in terms of overall accuracy for displacement fields with sufficiently high spatial frequency content, in addition to having higher spatial resolution. Finally, wOFA was demonstrated on two sets of experimental data to produce BOS images with higher spatial resolution and sensitivity than ILS, without the introduction of additional noise.

Based on the results presented here, along with results from other researchers on OFA methods (e.g. [15, 24]), it seems that OFA or wOFA are preferable to process BOS images compared to state-of-the-art ILS or cross-correlation-based methods, which have an inherent loss in spatial resolution that adversely impacts overall accuracy. The wOFA method presented here is mathematically stable without explicit regularization and computationally efficient. In future research efforts, it may be possible to automatically determine the optimal value of $\lambda$ without user input by using the known characteristics, e.g. spatial frequency, of the specified background pattern. Preliminary work using the 2D sinusoid background support the possibility that this will be the case, as under-regularized estimations of the displacement fields have noise content with a spectral signature related to the period of the sinusoid. In addition, as identified by Grauer and Steinberg [24], there is a similarity between the inverse problems of optical flow and tomography. Both are ill-posed inverse problems, and they can be solved simultaneously. Therefore, there are likely substantial benefits to extending wOFA to multiple dimensions, and unifying the optical flow and tomography calculations using a wavelet framework.

\section{Funding Sources}

The authors acknowledge the NASA Glenn Summer Faculty Fellowship Program for funding and support of this work.

\section{References}

[1] Dalziel, S. B., Hughes, G. O., and Sutherland, B. R., "Whole-field density measurements by 'synthetic schlieren'," Experiments in Fluids, Vol. 28, No. 4, 2000, pp. 322-335. https://doi.org/10.1007/s003480050391 
[2] Raffel, M., Richard, H., and Meier, G. E. A., "On the applicability of background oriented optical tomography for large scale aerodynamic investigations," Experiments in Fluids, Vol. 28, No. 5, 2000, pp. 477-481. https://doi.org/10.1007/s003480050408

[3] Raffel, M., "Background-oriented schlieren (BOS) techniques," Experiments in Fluids, Vol. 56, No. 3, 2015.

[4] Settles, G. S., and Hargather, M. J., "A review of recent developments in schlieren and shadowgraph techniques," Measurement Science and Technology, Vol. 28, 2017. https://doi.org/10.1088/1361-6501/aa5748.

[5] Venkatakrishnan, L., "Density Measurements in an Axisymmetric Underexpanded Jet by Background-Oriented Schlieren Technique,” AIAA Journal, Vol. 43, No. 7, 2005, pp. 1574-1579. https://doi.org/10.2514/1.12647

[6] Castner, R., "Exhaust Nozzle Plume Effects on Sonic Boom,” Journal of Aircraft, Vol. 49, No. 2, 2012 , pp. 415-422. https://doi.org/10.2514/1.c031305.

[7] Bauknecht, A., Merz, C. B., Raffel, M., Landolt, A., and Meier, A. H., "Blade-Tip Vortex Detection in Maneuvering Flight Using the Background-Oriented Schlieren Technique,” Journal of Aircraft, Vol. 51, No. 6, 2014, pp. $2005-2014$. https://doi.org/10.2514/1.c032672.

[8] Brown, C. A., Clem, M. M., and Fagan, A. F., "Investigation of Broadband Shock Noise from a Jet Near a Planar Surface," Journal of Aircraft, Vol. 52, No. 1, 2015, pp. 266-273. https://doi.org/10.2514/1.c032695

[9] Geerts, J. S., and Yu, K. H., "Systematic Application of Background-Oriented Schlieren for Isolator Shock Train Visualization," AIAA Journal, Vol. 55, No. 4, 2017, pp. 1105-1117. https://doi.org/10.2514/1.j054991.

[10] Raffel, M., Willert, C. E., Scarano, F., Kähler, C. J., Wereley, S. T., and Kompenhans, J., Particle image velocimetry: a practical guide, Springer, 2018.

[11] Pan, B., Qian, K., Xie, H., and Asundi, A., "Two-dimensional digital image correlation for in-plane displacement and strain measurement: a review," Measurement Science and Technology, Vol. 20, No. 6, 2009. https://doi.org/10.1088/0957$0233 / 20 / 6 / 062001$

[12] Adrian, R. J., “Twenty years of particle image velocimetry,” Experiments in Fluids, Vol. 39, 2005, pp. 159-169.

[13] Rajendran, L. K., Bane, S. P. M., and Vlachos, P. P., "Dot tracking methodology for background-oriented schlieren (BOS)," Experiments in Fluids, Vol. 60, No. 11, 2019. https://doi.org/10.1007/s00348-019-2793-3.

[14] Horn, B. K. P., and Schunck, B. G., “Determining Optical Flow,” Artificial Intelligence, Vol. 17, 1981, pp. $185-203$.

[15] Atcheson, B., Heidrich, W., and Ihrke, I., "An evaluation of optical flow algorithms for background oriented schlieren imaging," Experiments in Fluids, Vol. 46, No. 3, 2009, pp. 467-476.

[16] Lucas, B., and Kanade, T., "An iterative image registration technique with an application to stereo vision," Proceedings of the 7th international joint conference on artificial intelligence, 1981, pp. 674-679. 
[17] Brox, T., Bruhn, A., Papenberg, N., and Weickert, J., "High Accuracy Optical Flow Estimation Based on a Theory for Warping," European conference on computer vision, Springer, 2004, pp. 25-36. https://doi.org/10.1007/978-3-540-24673-2_3

[18] Atcheson, B., Ihrke, I., Heidrich, W., Tevs, A., Bradley, D., Magnor, M., and Seidel, H.-P., “Time-resolved 3D capture of nonstationary gas flows," ACM Transactions on Graphics, Vol. 27, No. 5, 2008, pp. 1-9. https://doi.org/10.1145/1409060.1409085

[19] Hayasaka, K., Tagawa, Y., Liu, T., and Kameda, M., "Optical-flow-based background-oriented schlieren technique for measuring a laser-induced underwater shock wave," Experiments in Fluids, Vol. 57, No. 12, 2016. https://doi.org/10.1007/s00348-016$2271-0$

[20] Hill, M. A., and Haering, E. A., "Ground-to-air flow visualization using Solar Calcium-K line Background-Oriented Schlieren," Experiments in Fluids, Vol. 58, No. 1, 2016. https://doi.org/10.1007/s00348-016-2285-7

[21] Smith, N. T., Durston, D., and Heineck, J. T., "Retroreflective Background-Oriented Schlieren Imaging Results from the NASA Ames Plume-Shock Interaction Test," 55th AIAA Aerospace Sciences Meeting, AIAA, 2017, p. 0043. https: //doi.org/10.2514/6.2017-0043

[22] Smith, N. T., Heineck, J. T., and Schairer, E. T., "Optical Flow for Flight and Wind Tunnel Background Oriented Schlieren Imaging," 55th AIAA Aerospace Sciences Meeting, AIAA, 2017, p. 0472. https://doi.org/10.2514/6.2017-0472

[23] Grauer, S. J., Unterberger, A., Rittler, A., Daun, K. J., Kempf, A. M., and Mohri, K., "Instantaneous 3D flame imaging by background-oriented schlieren tomography," Combustion and Flame, Vol. 196, 2018, pp. 284-299. https://doi.org/10.1016/j. combustflame.2018.06.022.

[24] Grauer, S. J., and Steinberg, A. M., "Fast and robust volumetric refractive index measurement by unified background-oriented schlieren tomography," Experiments in Fluids, Vol. 61, No. 3, 2020. https://doi.org/10.1007/s00348-020-2912-1

[25] Heineck, J. T., Banks, D. W., Smith, N. T., Schairer, E. T., Bean, P. S., and Robillos, T., "Background-Oriented Schlieren Imaging of Supersonic Aircraft in Flight,” AIAA Journal, 2020, pp. 1-11. https://doi.org/10.2514/1.j059495

[26] Zhang, X., Wang, L.-M., Liu, B., Luo, Y., and Han, X., "Hybrid Adaptive Wavelet-Based Optical Flow Algorithm for Background Oriented Schlieren (BOS) Experiments," Mathematical Problems in Engineering, Vol. 2020, 2020, pp. 1-15. https://doi.org/10.1155/2020/5138153

[27] Schmidt, B. E., and Sutton, J. A., "High-resolution velocimetry from tracer particle fields using a wavelet-based optical flow method," Experiments in Fluids, Vol. 60, No. 37, 2019. https://doi.org/10.1007/s00348-019-2685-6.

[28] Schmidt, B. E., and Sutton, J. A., "Improvements in the accuracy of wavelet-based optical flow velocimetry (wOFV) using an efficient and physically based implementation of velocity regularization," Experiments in Fluids, Vol. 61, No. 2, 2020. https://doi.org/10.1007/s00348-019-2869-0

[29] Black, M. J., and Anandan, P., "The robust estimation of multiple motions: parametric and piecewise-smooth flow fields," Computer Vision and Image Understanding, Vol. 63, No. 1, 1996, pp. 75-144. 
[30] Corpetti, T., Heitz, D., Arroyo, G., Mémin, E., and Santa-Cruz, A., "Fluid experimental flow estimation based on an optical-flow scheme," Experiments in Fluids, Vol. 40, No. 1, 2006, pp. 80-97. https://doi.org/10.1007/s00348-005-0048-y.

[31] Yuan, J., Schnörr, C., and Mémin, E., “Discrete Orthogonal Decomposition and Variational Fluid Flow Estimation,” Journal of Mathematical and Imaging Vision, Vol. 28, 2007, pp. 67-80.

[32] Kadri-Harouna, S., Dérian, P., Héas, P., and Mémin, E., “Divergence-free wavelets and high order regularization,” International Journal of Computer Vision, Vol. 103, No. 1, 2013, pp. 80-99. https://doi.org/10.1007/s11263-012-0595-7.

[33] Vogel, C. R., and Oman, M. E., "Fast, robust total variation-based reconstruction of noisy, blurred images," IEEE Transactions on Image Processing, Vol. 7, No. 6, 1998, pp. 813-824. https://doi.org/10.1109/83.679423

[34] Paris, S., Hasinoff, S. W., and Kautz, J., "Local Laplacian filters: Edge-aware image processing with a Laplacian pyramid." ACM Transactions on Graphics, 2011.

[35] Beylkin, G., "On the representation of operators in bases of compactly supported wavelets," SIAM Journal of Numerical Analysis, Vol. 6, No. 6, 1992, pp. 1716-1740.

[36] Nuttall, A., "Some windows with very good sidelobe behavior," IEEE Transactions on Acoustics, Speech, and Signal Processing, Vol. 29, No. 1, 1981, pp. 84-91. https://doi.org/10.1109/tassp.1981.1163506

[37] Ergin, F. G., "Dynamic masking techniques for particle image velocimetry," Journal of Thermal Science and Technology, Vol. 37, No. 2, 2017, pp. 61-74.

[38] Masullo, A., and Theunissen, R., "Automated mask generation for PIV image analysis based on pixel intensity statistics," Experiments in Fluids, Vol. 58, No. 6, 2017. https://doi.org/10.1007/s00348-017-2357-3

[39] Zitová, B., and Flusser, J., “Image registration methods: a survey,” Image and Vision Computing, Vol. 21, No. 11, 2003, pp. 977-1000. https://doi.org/10.1016/s0262-8856(03)00137-9

[40] Kiger, K. T., and Pan, C., "PIV Technique for the simultaneous measurement of dilute two-phase flows," Journal of Fluids Engineering, Vol. 122, 2000, pp. 811-818.

[41] Perlin, K., “An image synthesizer,” ACM SIGGRAPH Computer Graphics, Vol. 19, No. 3, 1985, pp. 287-296. https: //doi.org/10.1145/325165.325247

[42] Schmidt, B. E., and Sutton, J. A., "Evaluation of Gas- and Particle-Phase Separation Methods for Velocity Measurements in Turbulent Multiphase Flows," Experiments in Fluids, Vol. 61, No. 12, 2020. https://doi.org/10.1007/s00348-020-03082-7.

[43] Cook, R. L., and DeRose, T., “Wavelet noise,” ACM Transactions on Graphics, Vol. 24, No. 3, 2005 , pp. 803-811. https://doi.org/10.1145/1073204.1073264

[44] Settles, G. S., Schlieren and Shadowgraph Techniques, $1^{\text {st }}$ ed., Springer Berlin Heidelberg, 2001. 
[45] Cliff, S. E., Durston, D. A., Elmiligui, A. A., Walker, E. L., and Carter, M. B., "Experimental and Computational Sonic Boom Assessment of Lockheed-Martin N+2 Low Boom Models,” Tech. Rep. NASA/TP-2015-218483, NASA, 2015.

[46] Weisberger, J. M., Bathel, B. F., Jones, S. B., Woike, M. R., Ponder, J. D., Heineck, J. T., and Schairer, E. T., "Preparations for Tomographic Background-Oriented Schlieren Measurements in the 11-by 11-Foot Transonic Wind Tunnel," AIAA Aviation 2020 Forum, AIAA, 2020, p. 3102. https://doi.org/10.2514/6.2020-3102 